\title{
Non-universal critical behaviour of a mixed-spin Ising model on the extended Kagomé lattice*
}

\author{
J.Strečka, L.Čanová \\ Department of Theoretical Physics and Astrophysics, \\ Faculty of Science, P. J. Šafárik University, \\ Park Angelinum 9, 04001 Košice, Slovak Republic
}

Received July 18, 2005, in final form November 16, 2005

\begin{abstract}
The mixed spin-1/2 and spin-3/2 Ising model on the extended Kagomé lattice is solved by establishing a mapping correspondence with the eight-vertex model. When the parameter of uniaxial single-ion anisotropy tends to infinity, the model system becomes exactly solvable as the staggered eight-vertex model satisfying the free-fermion condition. The critical points within this manifold can be characterized by critical exponents from the standard Ising universality class. The critical points within another subspace of interaction parameters, which corresponds to a coexistence surface between two ordered phases, can be approximated by corresponding results of the uniform eight-vertex model satisfying the zero-field condition. This coexistence surface is bounded by a line of bicritical points that have non-universal continuously varying critical indices.
\end{abstract}

Key words: Ising model, eight-vertex model, bicritical points, non-universality

PACS: $75.10 . H k, 05.50 .+q, 75.40 . C x$

\section{Introduction}

Investigation of phase transitions and critical phenomena belongs to the most intensively studied topics in the equilibrium statistical physics. A considerable progress in the understanding of orderdisorder phenomena has been achieved by solving planar Ising models that represent valuable exceptions of exactly solvable lattice-statistical models with a non-trivial critical behaviour [1]. Although phase transitions of planar Ising models have already been understood in many respects, there are still a lot of obscurities connected with a criticality of more complicated spin systems exhibiting reentrant transitions, non-universal critical behaviour, tricritical phenomena, etc. It is worth mentioning, however, that several complicated Ising models can be exactly treated by transforming them to the solvable vertex models. A spin-1/2 Ising model on the union jack (centered square) lattice, which represents a first exactly solvable system exhibiting reentrant transitions [2], can be for instance reformulated as a free-fermion eight-vertex model [3]. It should be also pointed out that an equivalence with the vertex models has already provided a precise confirmation of the reentrant phenomenon in the anisotropic spin-1/2 Ising models on extended Kagomé lattice [4] and centered honeycomb lattice [5] as well.

Despite the significant amount of effort, there are only a few exactly solvable Ising models consisting of mixed spins of different magnitudes, which are usually called as mixed-spin Ising models. A strong scientific interest focused on the mixed-spin systems arises partly due to a much richer critical behaviour they display compared with their single-spin counterparts and partly due to the fact that they represent the most simple models of ferrimagnets having a wide potential applicability in practice. Using the extended versions of decoration-iteration and star-triangle mapping transformations, Fisher [6] has derived exact solutions of the mixed spin- $1 / 2$ and spin- $S(S \geqslant 1)$

*This work was financially supported under the grants VEGA 1/2009/05 and APVT 20-005204. 
Ising models on the honeycomb, diced and decorated honeycomb lattices. Notice that these mapping transformations were later on further generalized in order to also account for the single-ion anisotropy effect. The effect of uniaxial and biaxial single-ion anisotropies has been precisely investigated on the mixed-spin honeycomb lattice [7] as well as on some decorated planar lattices [8]. With the exception of several mixed-spin models formulated on the Bethe (Cayley tree) lattices, which can be accurately treated within a discrete non-linear map [9] or exact recursion equations [10], these are the only mixed-spin planar Ising models with generally known exact solutions, yet.

One of the most outstanding findings emerging in the phase transition theory is a non-universal critical behaviour of some planar Ising models, which is in obvious contradiction with the idea of universality hypothesis [11]. The mixed spin-1/2 and spin- $S$ Ising model on the union jack lattice [12] represents very interesting system from this viewpoint as it exhibits a remarkable line of bicritical points that have continuously varying critical indices obeying the weak universality hypothesis [13]. In the present article, we shall investigate a topologically similar mixed spin- $1 / 2$ and spin$3 / 2$ Ising model on the extended Kagomé lattice by establishing a mapping correspondence with the staggered and uniform eight-vertex models, respectively. In a certain subspace of interaction parameters, the model under investigation becomes exactly solvable as the staggered eight-vertex model satisfying the free-fermion condition [14]. Even if a non-validity of the free-fermion condition in the rest of parameter space is simply ignored, one still obtains a rather reliable estimate of the criticality within free-fermion approximation [15]. Finally, the critical points within another subspace of interaction parameters can be approximated from the relevant solution of the uniform eight-vertex model satisfying the zero-field condition.

The outline of this paper is as follows. In section 2, a detailed formulation of the model is presented and subsequently, the mapping correspondence that ensures an equivalence with the eight-vertex models will be derived. The most interesting numerical results for a critical behaviour will be presented and particularly discussed in section 3. Finally, some concluding remarks are drawn in section 4 .

\section{Formulation}

Let us begin with considering the mixed spin-1/2 and spin-3/2 Ising model on the extended Kagomé lattice $\mathcal{L}$ schematically illustrated in figure 1. The mixed-spin Kagomé lattice consists of the spin-1/2 (empty) and spin-3/2 (filled circles) atoms placed on the six- and four-coordinated sites, respectively. The total Hamiltonian defined upon the underlying lattice $\mathcal{L}$ reads:

$$
\mathcal{H}_{\text {mix }}=-J \sum_{(i, j) \subset \mathcal{J}}^{2 N} S_{i} \sigma_{j}-J^{\prime} \sum_{(k, l) \subset \mathcal{K}}^{2 N} \sigma_{k} \sigma_{l}-D \sum_{i=1}^{N / 2} S_{i}^{2},
$$

where $\sigma_{j}= \pm 1 / 2$ and $S_{i}= \pm 1 / 2, \pm 3 / 2$ are Ising spin variables, $J$ denotes the exchange interaction between nearest-neighbouring spin- $1 / 2$ and spin-3/2 pairs and $J^{\prime}$ labels the interaction between the spin-1/2 pairs that are next-nearest-neighbours on the extended Kagomé lattice $\mathcal{L}$. Finally, the parameter $D$ measures a strength of the uniaxial single-ion anisotropy acting on the spin- $3 / 2$ sites and $N$ denotes the total number of the spin- $1 / 2$ sites.

In order to proceed further with calculation, the central spin-3/2 atoms should be firstly decimated from all faces of extended Kagomé lattice $\mathcal{L}$. After the decimation, i.e. after performing a summation over spin degrees of freedom of the spin-3/2 sites (filled circles), the partition function of the mixed-spin system can be rewritten as:

$$
\mathcal{Z}_{\text {mix }}=\sum_{\{\sigma\}} \prod_{m=1}^{N / 2} \omega_{m}^{\mathcal{A}}\left(\sigma_{i}, \sigma_{j}, \sigma_{k}, \sigma_{l}\right) \prod_{n=1}^{N / 2} \omega_{n}^{\mathcal{B}}\left(\sigma_{i}, \sigma_{j}, \sigma_{k}, \sigma_{l}\right)
$$

Above, the summation is performed over all possible spin configurations available at the spin- $1 / 2$ sites and the first (second) product is over $N / 2$ faces having four spin- $1 / 2$ sites $\sigma_{i}, \sigma_{j}, \sigma_{k}, \sigma_{l}$ placed in the corners of square plaquettes with (without) a central spin- $3 / 2$ site in the middle of these 


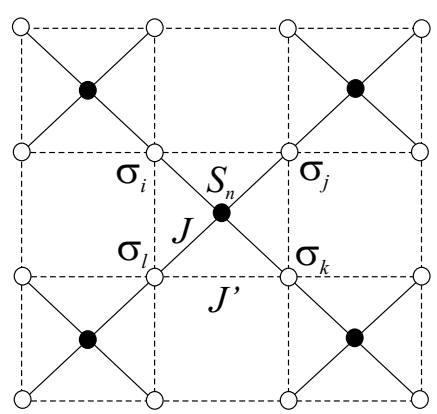

Figure 1. Diagrammatic representation of the extended Kagomé lattice composed of the mixed spin-1/2 (empty circles) and spin-3/2 (filled circles) sites, respectively. The solid (broken) lines depict the nearest-neighbour (next-nearest-neighbour) interactions.

plaquettes (see figure 1). The Boltzmann factors $\omega^{\mathcal{A}}(a, b, c, d)$ and $\omega^{\mathcal{B}}(a, b, c, d)$ assigned to two different kinds of alternating faces, which constitute the checkerboard lattice, can be defined as:

$$
\begin{aligned}
\omega^{\mathcal{A}}(a, b, c, d)= & 2 \exp \left[K^{\prime}(a b+b c+c d+d a) / 2+\Delta / 4\right] \\
& \times\{\exp (2 \Delta) \cosh [3 K(a+b+c+d) / 2]+\cosh [K(a+b+c+d) / 2]\}, \\
\omega^{\mathcal{B}}(a, b, c, d)= & \exp \left[K^{\prime}(a b+b c+c d+d a) / 2\right],
\end{aligned}
$$

where $K=J /\left(k_{\mathrm{B}} T\right), K^{\prime}=J^{\prime} /\left(k_{\mathrm{B}} T\right), \Delta=D /\left(k_{\mathrm{B}} T\right), k_{\mathrm{B}}$ is Boltzmann's constant, and $T$ stands for the absolute temperature.

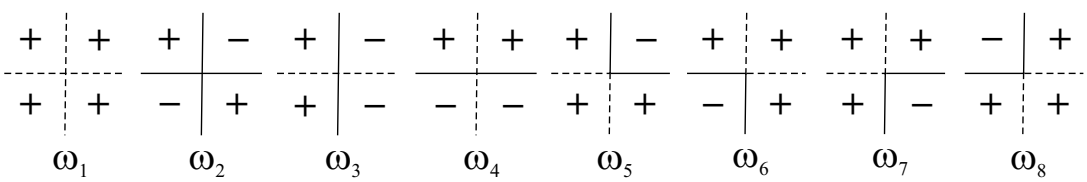

Figure 2. The eight possible line arrangements at a vertex of the dual lattice.

At this stage, the model under investigation can be rather straightforwardly mapped onto the staggered eight-vertex model defined on a dual checkerboard lattice $\mathcal{L}_{\mathcal{D}}$, since Boltzmann factors $\omega^{\mathcal{A}}(a, b, c, d)$ and $\omega^{\mathcal{B}}(a, b, c, d)$ are being invariant under the reversal of all four spin variables. Actually, there are maximally eight different spin arrangements giving different Boltzmann weights $\omega^{\mathcal{A}}(a, b, c, d)$ and $\omega^{\mathcal{B}}(a, b, c, d)$ for each kind of face. Diagrammatic representation of eight possible spin arrangements and their corresponding line coverings of the eight-vertex model is shown in figure 2. If, and only if, the adjacent spins are aligned opposite to each other, then solid lines are drawn on the edges of the dual lattice $\mathcal{L}_{\mathcal{D}}$, otherwise they are drawn as broken lines. It can be easily understood that eight possible line coverings around each vertex of the dual checkerboard lattice always correspond to two spin configurations, one is being obtained from the other by reversing all spins. Since there is an even number of solid (broken) lines incident to each vertex of the dual lattice $\mathcal{L}_{\mathcal{D}}$, the model becomes equivalent to the staggered eight-vertex model.

The Boltzmann weights $\omega^{\mathcal{A}}(a, b, c, d)$ and $\omega^{\mathcal{B}}(a, b, c, d)$, which correspond to eight possible line coverings emerging at vertices of the dual checkerboard lattice, can directly be calculated from equation (3):

$$
\begin{aligned}
& \omega_{1}^{\mathcal{A}}=2 \exp \left(K^{\prime} / 2+\Delta / 4\right)[\exp (2 \Delta) \cosh (3 K)+\cosh (K)] \\
& \omega_{2}^{\mathcal{A}}=2 \exp \left(-K^{\prime} / 2+\Delta / 4\right)[\exp (2 \Delta)+1], \\
& \omega_{3}^{\mathcal{A}}=\omega_{4}^{\mathcal{A}}=2 \exp (\Delta / 4)[\exp (2 \Delta)+1], \\
& \omega_{5}^{\mathcal{A}}=\omega_{6}^{\mathcal{A}}=\omega_{7}^{\mathcal{A}}=\omega_{8}^{\mathcal{A}}=2 \exp (\Delta / 4)[\exp (2 \Delta) \cosh (3 K / 2)+\cosh (K / 2)] ;
\end{aligned}
$$




$$
\begin{aligned}
& \omega_{1}^{\mathcal{B}}=\exp \left(K^{\prime} / 2\right), \quad \omega_{2}^{\mathcal{B}}=\exp \left(-K^{\prime} / 2\right), \\
& \omega_{3}^{\mathcal{B}}=\omega_{4}^{\mathcal{B}}=\omega_{5}^{\mathcal{B}}=\omega_{6}^{\mathcal{B}}=\omega_{7}^{\mathcal{B}}=\omega_{8}^{\mathcal{B}}=1 .
\end{aligned}
$$

Unfortunately, there does not exist a general exact solution to the staggered eight-vertex model with arbitrary Boltzmann weights $\omega_{i}^{\mathcal{A}}$ and $\omega_{j}^{\mathcal{B}}(i, j=1-8)$. However, if the weights (4) and (5) satisfy the so-called free-fermion condition:

$$
\Omega_{1} \Omega_{2}+\Omega_{3} \Omega_{4}=\Omega_{5} \Omega_{6}+\Omega_{7} \Omega_{8},
$$

the staggered eight-vertex model then becomes exactly solvable as the free-fermion model solved several years ago by Hsue, Lin and $\mathrm{Wu}$ [14]. The expressions which enter into the free-fermion condition (6) can be defined through:

$$
\begin{array}{rlrl}
\Omega_{1} & =\omega_{1}^{\mathcal{A}} \omega_{1}^{\mathcal{B}}+\omega_{2}^{\mathcal{A}} \omega_{2}^{\mathcal{B}}, & \Omega_{2}=\omega_{3}^{\mathcal{A}} \omega_{3}^{\mathcal{B}}+\omega_{4}^{\mathcal{A}} \omega_{4}^{\mathcal{B}}, \\
\Omega_{3} & =\omega_{5}^{\mathcal{A}} \omega_{6}^{\mathcal{B}}+\omega_{5}^{\mathcal{B}} \omega_{6}^{\mathcal{A}}, & \Omega_{4}=\omega_{7}^{\mathcal{A}} \omega_{8}^{\mathcal{B}}+\omega_{7}^{\mathcal{B}} \omega_{8}^{\mathcal{A}}, \\
\Omega_{5} \Omega_{6} & =\omega_{1}^{\mathcal{A}} \omega_{1}^{\mathcal{B}} \omega_{3}^{\mathcal{A}} \omega_{3}^{\mathcal{B}}+\omega_{2}^{\mathcal{A}} \omega_{2}^{\mathcal{B}} \omega_{4}^{\mathcal{A}} \omega_{4}^{\mathcal{B}}+\omega_{5}^{\mathcal{A}} \omega_{6}^{\mathcal{B}} \omega_{7}^{\mathcal{A}} \omega_{8}^{\mathcal{B}}+\omega_{5}^{\mathcal{B}} \omega_{6}^{\mathcal{A}} \omega_{7}^{\mathcal{B}} \omega_{8}^{\mathcal{A}}, \\
\Omega_{7} \Omega_{8} & =\omega_{1}^{\mathcal{A}} \omega_{1}^{\mathcal{B}} \omega_{4}^{\mathcal{A}} \omega_{4}^{\mathcal{B}}+\omega_{2}^{\mathcal{A}} \omega_{2}^{\mathcal{B}} \omega_{3}^{\mathcal{A}} \omega_{3}^{\mathcal{B}}+\omega_{5}^{\mathcal{A}} \omega_{6}^{\mathcal{B}} \omega_{7}^{\mathcal{B}} \omega_{8}^{\mathcal{A}}+\omega_{5}^{\mathcal{B}} \omega_{6}^{\mathcal{A}} \omega_{7}^{\mathcal{A}} \omega_{8}^{\mathcal{B}} .
\end{array}
$$

It can be readily proved that the free-fermion condition (6) holds in our case just as $D \rightarrow \pm \infty$, or $T \rightarrow \infty$. The restriction to infinitely strong single-ion anisotropy consequently leads to the familiar phase transitions from the standard Ising universality class, because in this case our model effectively reduces to a simple spin-1/2 Ising model on the extended Kagomé lattice. Within the manifold given by the constraint (6), the free-fermion model becomes critical as long as:

$$
\Omega_{1}+\Omega_{2}+\Omega_{3}+\Omega_{4}=2 \max \left\{\Omega_{1}, \Omega_{2}, \Omega_{3}, \Omega_{4}\right\} .
$$

It is noteworthy, however, that the critical condition (8) yields a rather reliable estimate of the criticality within the so-called free-fermion approximation [15] even if a non-validity of the freefermion condition (6) is simply ignored.

Now, we shall establish an approximate mapping between the staggered and uniform eightvertex models, since the second branch of exact solution is available just for the latter model under the zero-field condition [1]. For this purpose, let us define average Boltzmann weights of the staggered eight-vertex model, which would approximately transform the staggered eight-vertex model into the uniform one:

$$
\tilde{\omega}_{i}=\omega_{i}^{\mathcal{A}} \omega_{i}^{\mathcal{B}}, \quad(i=1-8)
$$

Note that the uniform eight-vertex model satisfies the zero-field condition just when its Boltzmann weights are pairwise and symmetrically equal to each other:

$$
\tilde{\omega}_{1}=\tilde{\omega}_{2}, \quad \tilde{\omega}_{3}=\tilde{\omega}_{4}, \quad \tilde{\omega}_{5}=\tilde{\omega}_{6}, \quad \tilde{\omega}_{7}=\tilde{\omega}_{8} .
$$

As we already have $\tilde{\omega}_{3}=\tilde{\omega}_{4}, \tilde{\omega}_{5}=\tilde{\omega}_{6}$, and $\tilde{\omega}_{7}=\tilde{\omega}_{8}$, the zero-field case is consequently reached by imposing the condition $\tilde{\omega}_{1}=\tilde{\omega}_{2}$ only, or equivalently:

$$
\exp (2 \Delta)=\frac{\exp \left(-2 K^{\prime}\right)-\cosh (K)}{\cosh (3 K)-\exp \left(-2 K^{\prime}\right)}
$$

According to Baxter's exact solution [1], the zero-field eight-vertex model becomes critical on the manifold (10) if:

$$
\tilde{\omega}_{1}+\tilde{\omega}_{3}+\tilde{\omega}_{5}+\tilde{\omega}_{7}=2 \max \left\{\tilde{\omega}_{1}, \tilde{\omega}_{3}, \tilde{\omega}_{5}, \tilde{\omega}_{7}\right\}
$$

It is easy to check that in our case $\tilde{\omega}_{1}$ represents the largest Boltzmann weight. Thus, the condition determining the criticality can also be written in this equivalent form:

$$
\begin{aligned}
& \exp \left(K_{\mathrm{c}}^{\prime}\right)\left[\exp \left(2 \Delta_{\mathrm{c}}\right) \cosh \left(3 K_{\mathrm{c}}\right)+\cosh \left(K_{\mathrm{c}}\right)\right]= \\
& =1+\exp \left(2 \Delta_{\mathrm{c}}\right)+2 \exp \left(2 \Delta_{\mathrm{c}}\right) \cosh \left(3 K_{\mathrm{c}} / 2\right)+2 \cosh \left(K_{\mathrm{c}} / 2\right),
\end{aligned}
$$


where $K_{\mathrm{c}}=J /\left(k_{\mathrm{B}} T_{\mathrm{c}}\right), K_{\mathrm{c}}^{\prime}=J^{\prime} /\left(k_{\mathrm{B}} T_{\mathrm{c}}\right), \Delta_{\mathrm{c}}=D /\left(k_{\mathrm{B}} T_{\mathrm{c}}\right)$, and $T_{\mathrm{c}}$ denotes the critical temperature. It should be stressed, nevertheless, that the critical exponents (with the exception of $\delta$ and $\eta$ ) describing a phase transition of the zero-field eight-vertex model depend on the function $\mu=$ $2 \arctan \left(\tilde{\omega}_{5} \tilde{\omega}_{7} / \tilde{\omega}_{1} \tilde{\omega}_{3}\right)^{1 / 2}$, in fact:

$$
\alpha=\alpha^{\prime}=2-\frac{\pi}{\mu}, \quad \beta=\frac{\pi}{16 \mu}, \quad \nu=\nu^{\prime}=\frac{\pi}{2 \mu}, \quad \gamma=\frac{7 \pi}{8 \mu}, \quad \delta=15, \quad \eta=\frac{1}{4} .
$$

Finally, let us explicitly evaluate the critical exponent $\beta$ that determines the disappearance of the spontaneous order as the critical temperature is approached from below:

$$
\beta^{-1}=\frac{32}{\pi} \arctan \left\{\frac{\exp \left(2 \Delta_{\mathrm{c}}\right) \cosh \left(3 K_{\mathrm{c}} / 2\right)+\cosh \left(K_{\mathrm{c}} / 2\right)}{\left[\exp \left(2 \Delta_{\mathrm{c}}\right)+1\right]^{3 / 4}\left[\exp \left(2 \Delta_{\mathrm{c}}\right) \cosh \left(3 K_{\mathrm{c}}\right)+\cosh \left(K_{\mathrm{c}}\right)\right]^{1 / 4}}\right\} .
$$

\section{Results and discussion}

Now, let us turn our attention to a discussion of the most interesting results obtained for the ground-state and finite-temperature phase diagrams. Solid lines displayed in figure 3 represent ground-state phase boundaries separating four distinct long-range ordered phases that emerge in the ground state when $J>0$. Spin order drawn in broken rectangles shows a typical spin configuration within basic unit cell of each phase. As could be expected, a sufficiently strong antiferromagnetic next-nearest-neighbour interaction $J^{\prime}$ alters the structure of the ground state owing to a competing effect with the nearest-neighbour interaction $J$. Due to a competition between the interactions, the central spins are free to flip within the phases III and IV and thus, these phases exhibit a remarkable coexistence of the order and the disorder. At last, it is worthwhile mentioning that a broken line connecting both triple points depicts a projection of the approximate critical line (13) into the $J^{\prime}-D$ plane. As this projection crosses zero-temperature plane along the ground-state transition line $D / J=-3 / 2-J^{\prime} / J$ between the phases I and IV, it is quite reasonable to suspect that this line represents a location of phase transitions between these phases.

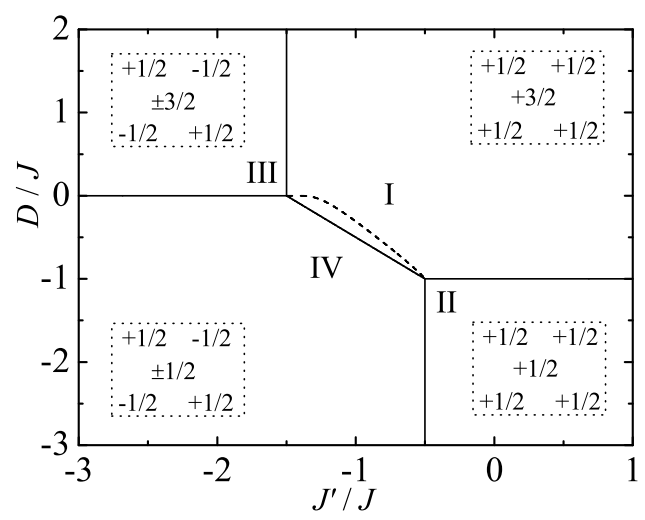

Figure 3. Ground-state phase diagram in the $J^{\prime}-D$ plane when $J>0$. Broken rectangles schematically illustrate a typical spin configuration within each phase. Broken line connecting both triple points shows a projection of the critical line (13) into the $J^{\prime}-D$ plane.

Let us more deeply investigate this line of critical points. The critical temperatures calculated from the uniform zero-field eight-vertex model must simultaneously obey both the zero-field condition (11) and the critical condition (13). It is easy to check that the former condition necessitates $-1.5<J^{\prime} / J<-0.5$ and $-1.0<D / J<0.0$. Figure 4 (a) displays a projection of this critical line into the $J^{\prime}-T_{\text {c }}$ plane (the dependence scaled to the left axis) and respectively, a projection into the $J^{\prime}-D$ plane which is scaled to the right axis. Along this critical line, the critical exponents are expected to vary with interaction parameters as they have to follow the equations (14). For illustration, figure 4(b) shows how the critical index $\beta$ changes along the critical line. Apparently, 

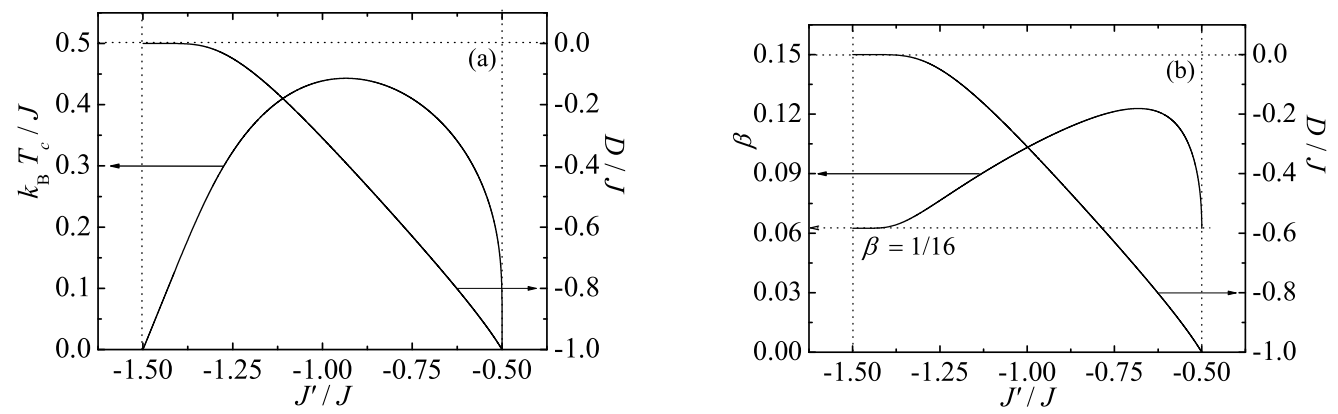

Figure 4. (a) The curve scaled to the left axis shows how the critical temperature changes with a strength of the next-nearest-neighbour coupling $J^{\prime} / J$, the curve scaled to the right axis depicts a variation of the single-ion anisotropy parameter along this line; (b) The same as for figure 4(a), but the critical exponent $\beta$ is now scaled to the left axis. In both figures broken lines are guides for eyes only.

the exponent $\beta$ approaches its smallest possible value $1 / 16$ by reaching both triple points with zero critical temperature. However, it is also quite interesting to ascertain that its greatest value is below the value $1 / 8$ that predicts the universality hypothesis for planar Ising systems [1].

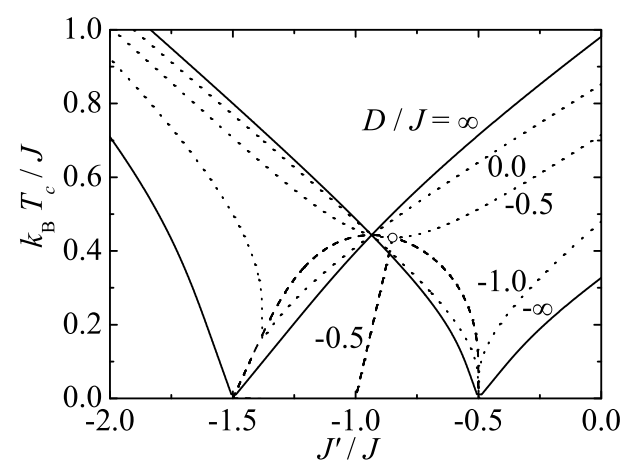

Figure 5. A plot critical temperature versus the ratio $J^{\prime} / J$ for several values of the single-ion anisotropy term $D / J$. For details see the text.

Before concluding, few remarks should be addressed to a global finite-temperature phase diagram plotted in figure 5 , which displays the critical temperature as a function of the ratio $J^{\prime} / J$ for several values of the single-ion anisotropy $D / J$. Critical boundaries depicted as solid lines represent exact critical points obtained from the free-fermion solution (8) of the staggered eight-vertex model obtained under the constraint (6), which is fulfilled in the limiting cases $D / J \rightarrow \pm \infty$. Dotted critical lines show the estimated critical temperatures calculated from the free-fermion approximation simply ignoring the non-validity of the free-fermion condition (6) for any finite value of $D / J$. Approximative solution related to the critical points (13) of the uniform zero-field eight-vertex model on the variety (11) is displayed as a rounded broken line. It is quite obvious from the ground-state phase diagram (figure 3) that a right (left) wing of the displayed critical boundaries corresponds to the phase I (III) if $D / J>0.0$, while it corresponds to the phase II (IV) if $D / J<-1.0$. Actually, both the exact and the approximate critical points resulting from the free-fermion solution correctly reproduce the ground-state boundaries between these phases. When the single-ion anisotropy parameter is selected within the range $-1.0<D / J<0.0$ (see for instance the curve for $D / J=-0.5$ ), however, the critical line obtained from the free-fermion approximation meets at a bicritical (circled) point with the critical line of the equivalent uniform zero-field eight-vertex model as it has been already reasoned by Lipowski and Horiguchi [12] who have solved similar spin system on the union-jack lattice. In such a case, the right and left part (with respect to the bicritical point) of this critical line separate the phases I and IV, respectively, 
and a line of first-order phase transitions is expected to terminate at this special multicritical point. There are strong indications supporting this concept [12], actually, the almost straight broken line depicting the zero-field condition (11) should always show the coexistence of these two phases as it starts from the point that determines their coexistence in the ground state. With regard to the aforementioned arguments one may conclude that the coexistence surface between the phases I and IV lies inside the area, which is bounded by the line of bicritical points (rounded broken line) having the non-universal interaction-dependent critical exponents.

Finally, we should remark a feasible appearance of reentrant transitions which can be observed in the critical lines near the coexistence points $D / J=0.0$ and -1.0 . It is quite apparent that the observed reentrance can be explained in terms of the coexistence of a partial order and partial disorder emerging in the both high-temperature reentrant phases III and IV. As a matter of fact, the partial disorder of the spin-3/2 atoms can compensate a loss of entropy that occurs in these phases due to a thermally induced partial ordering of the spin- $1 / 2$ atoms what is in a good accordance with the necessary condition conjectured for the appearance of reentrant phase transitions $[4,5]$.

\section{Concluding remarks}

The work reported in the present article provides a relatively precise information on the critical behaviour of the mixed spin- $1 / 2$ and spin-3/2 Ising model on the extended Kagomé lattice by establishing a mapping correspondence with the staggered and uniform eight-vertex models, respectively. The main focus of the present work has been aimed at the examination of the criticality depending on the single-ion anisotropy strength as well as on the strength of the competing next-nearest-neighbour interaction. The location of the critical boundaries has accurately been determined from the free-fermion solution of the staggered eight-vertex model and the zero-field solution of the uniform eight-vertex model, respectively, whereas the validity of both mappings is restricted to certain subspaces of interaction parameters only. In the rest of parameter space, the free-fermion approximation has been used to estimate the critical boundaries as this method should provide a meaningful approximation giving a rather reliable estimate to the true transition temperatures.

The remarkable critical line consisting of bicritical points, which bounds the coexistence surface between two long-range ordered phases is of theoretical interest in this model. The bicritical points can be characterized by non-universal interaction-dependent critical exponents that satisfy the weak universality hypothesis. Moreover, the same arguments as those suggested by Lipowski and Horiguchi [12] have enabled us to identify the zero-field condition (11) with the location of the first-order transition lines separating these two ordered phases.

It should be remarked that the spin system considered also shows reentrant phase transitions on account of the competition between the nearest- and next-nearest-neighbour interactions. Our results are in agreement with the conjecture [4] stating that the reentrance appears as a consequence of the coexistence of a partial order and disorder, namely, the partial disorder induced among spin$3 / 2$ atoms can compensate for the loss of entropy, which occurs on behalf of the partial ordering of the spin- $1 / 2$ atoms in the both high-temperature partially ordered phases. 


\title{
References
}

1. Baxter R.J., Ann. Phys., 1972, 70, 193; Baxter R.J., Proc. R. Soc. Lond. A, 1986, 404, 1; Baxter R.J. Exactly Solved Models in Statistical Mechanics. Academic Press, New York, 1982.

2. Vaks V., Larkin A., Ovchinnikov Yu.N., Sov. Phys. JETP, 1966, 22, 820.

3. Wu F.Y., Lin K.Y., J. Phys. A: Math. Gen., 1987, 20, 5737.

4. Azaria P., Diep H.T., Giacomini H., Phys. Rev. Lett., 1987, 59, 1629; Debauche M., Diep H.T., Azaria P., Giacomini H., Phys. Rev. B, 1991, 44, 2369.

5. Diep H.T., Debauche M., Giacomini H., Phys. Rev. B, 1991, 43, 8759; Diep H.T., Debauche M., Giacomini H., J. Magn. Magn. Mater., 1992, 104-107, 184.

6. Fisher M.E., Phys. Rev., 1959, 113, 969; Syozi I. Phase Transitions and Critical Phenomena, vol. 1, eds. Domb C. and Green M.S. Academic, New York, 1972.

7. Gonçalves L.L., Phys. Scripta, 1985, 32, 248; Gonçalves L.L., Phys. Scripta, 1986, 33, 192; Tucker J.W., J. Magn. Magn. Mater., 1999, 95, 133; Dakhama A., Benayad N., J. Magn. Magn. Mater., 2000, 231, 117; Strečka J., Jaščur M., Acta Electrotechnica et Informatica, 2002, 2, 102; Strečka J., Jaščur M., Phys. Rev. B, 2004, 70, 014404; Jaščur M., Strečka J., Physica A, 2005, 358, 393.

8. Jaščur M., Physica A, 1998, 252, 217; Dakhama A., Physica A, 1998, 252, 225; Lacková S., Jaščur M., Acta Phys. Slovaca, 1998, 48, 623.

9. da Silva N.R., Salinas S.R., Phys. Rev. B, 1991, 44, 852.

10. Albayrak E., Keskin M., J. Magn. Magn. Mater., 2003, 261, 196; Albayrak E., Int. J. Mod. Phys. B, 2004, 18, 3959; Albayrak E., Alci A., Physica A, 2005, 345, 48; Ekiz C., Physica A, 2005, 347, 353; Ekiz C., Physica A, 2005, 353, 286.

11. Griffiths R.B., Phys. Rev. Lett., 1970, 24, 1479.

12. Lipowski A., Horiguchi T., J. Phys. A: Math. Gen., 1995, 28, L261; Strečka J., Phys. Stat. Sol. B, in press.

13. Suzuki M., Progr. Theor. Phys., 1974, 51, 1992.

14. Hsue C.S., Lin K.Y., Wu F.Y., Phys. Rev. B, 1975, 12, 429.

15. Fan C., Wu F. Y., Phys. Rev., 1969, 179, 560; Fan C., Wu F. Y., Phys. Rev. B, 1970, $2,723$.

\section{Неуніверсальна критична поведінкамоделі Ізінга зі змішаними спінами на розширеній гратці Кагоме}

\author{
Й.Стречка, Л.Чанова \\ Відділ теоретичної фізики та астрофізики, \\ факультет науки, Університет ім. П.Й.Шафаріка, \\ 04001 Кошіце, Словацька Республіка \\ Отримано 18 липня 2005 р., в остаточному вигляді - \\ 16 листопада 2005 р.
}

\begin{abstract}
Модель Ізінга зі змішаними 1/2 та 3/2 спінами на розширеній гратці Кагоме розв'язується шляхом встановлення відповідності з восьмивершинною моделлю. Коли параметр одновісної анізотропії окремого іона прямує до безмежності, тоді модельна система стає точно розв'язуваною як шахматна восьмивершинна модель, що задовольняє умові вільних ферміонів. Критичні точки для цієї множини можуть бути охарактеризовані критичними експонентами з стандартного класу ізігновської універсальності. Критичні точки в межах іншого підпростору параметрів взаємодії, які відповідають поверхні між двома співіснуючими упорядкованими фазами, можуть бути апроксимовані відповідними результатами для однорідної восьмивершинної моделі, що задовольняє умові нульового поля. Ця поверхня співіснування є пов'язана з лінією бікритичних точок, що мають неуніверсальні постійно змінні критичні індекси.
\end{abstract}

Ключові слова: модель ґінга, восьмивершинна модель, бікритичні точки, неуніверсальність

PACS: $75.10 . H k, 05.50 .+q, 75.40 . C x$ 